\title{
VIABILIDADE ECONÔMICO-FINANCEIRA DE UM PROJETO DE PRODUÇÃO DE ARROZ NA CIDADE DE AGUDO - RS
}

\author{
Paula Donaduzzi Rigo ${ }^{1}$ \\ Patrícia Schrippe ${ }^{2}$ \\ Flaviani Souto Bolzan Medeiros ${ }^{3}$ \\ Andreas Dittmar Weise ${ }^{4}$
}

\begin{abstract}
Resumo: O arroz é essencial para a nutrição humana, sendo que é fonte de carboidratos e proteínas. O Rio Grande do Sul conta com a maior produção de arroz irrigado do Brasil, dentro deste, a região da Quarta Colônia, região central do Estado, vem apresentando ao longo dos anos uma alta produtividade por hectare. Nesse contexto, o presente estudo objetiva analisar a viabilidade econômico-financeira de se produzir arroz, como pequeno e médio agricultor, na cidade de Agudo - RS. Para este objetivo é indispensável utilizar métodos que compõe o escopo da disciplina de Engenharia econômica: o método de Valor Presente Líquido (VPL) e o Tempo de retorno de investimento descontado (Payback descontado). Metodologicamente, o trabalho foi realizado por meio de um estudo de caso em uma propriedade localizada em Agudo, com acompanhamento da safra 2013/2014, como uma pesquisa quantitativa, do tipo descritiva no que se refere aos objetivos. Dessa maneira, os métodos aplicados resultaram na inviabilidade econômico-financeira de se produzir arroz, sendo que o Payback descontado em 25 anos ainda não apresentou retorno do investimento. Para somar à inviabilidade, o VPL calculado foi de - R \$ 892.402,33. Entretanto, a produção de arroz é extremamente importante para todos os setores da sociedade, e com isso, se observam necessárias políticas públicas que garantam que a produção de arroz seja possível e segura.
\end{abstract}

Palavras-Chave: Produção de arroz; Engenharia econômica; Valor Presente Líquido; Payback descontado.

\begin{abstract}
Rice is essential of the human nutrition, it is a source of carbohydrates and proteins. Rio Grande do Sul has the first place in the production of irrigated rice in Brazil, within this, the Quarta Colônia has shown over the years a high productivity per hectare. On this context, the present study aims to analyze the economic viability of producing rice, by small and medium farmers. For this purpose it is essential to use methods from the discipline of economics engineering: the Method of Net Present Value (NPV) and the payback time of the investment discounted (Payback discounted). Methodologically, this study was carried out through a case study on a property located in Agudo, by monitoring of the 2013/2014 grain harvest season, as a quantitative research, descriptive referring to the goals. Thus, the method used resulted in the economic impossibility to produce rice, and the Payback discounted not shows return of investment in 25 years yet. And to add the impracticability, the NPV resulted in - R\$ 893,130.60. However, rice production is extremely important to all sectors of society, and thus, it needs public policies to ensure that rice production are possible and safe.
\end{abstract}

Keywords: Rice production; economic engineering; Net present value; Payback discounted.

\footnotetext{
${ }^{1}$ Universidade Federal de Santa Maria. E-mail: pauladonaduzzi@ gmail.com

2 PPGEP/UFSM - Pós-graduação em Engenharia de Produção / Universidade Federal de Santa Maria. Email: pschrippe@gmail.com

${ }^{3}$ PPGEP/UFSM -Pós-graduação em Engenharia de Produção / Universidade Federal de Santa Maria. E-mail: flaviani.13@gmail.com

4 PPGEP/UFSM -Pós-graduação em Engenharia de Produção / Universidade Federal de Santa Maria. Email: mail@weise.de
} 


\section{INTRODUÇÃO}

O arroz é fonte de proteínas e carboidratos, e se encontra presente como o protagonista da refeição diária do brasileiro. O Brasil produz, em média, 11 milhões de toneladas de arroz e está entre os principais produtores do mundo, sendo que o grande contribuinte é o Estado do Rio Grande do Sul, com uma produção representativa superior a $50 \%$ do total produzido no país (EMBRAPA, 2014a).

As lavouras de produção de arroz da região da Quarta Colônia, localizadas na região central do Estado do Rio Grande do Sul, apresentaram nos últimos anos uma produtividade média de 7.000 $\mathrm{kg}$ por hectare, sendo que, a região é favorável para alcançar até $10.000 \mathrm{~kg}$ por hectare (Diário de Santa Maria, 2013). Gelatti, 2014 ressalta que a cidade de Agudo, pertencente à Quarta Colônia, segue a mesma linha de produtividade e em relação à quantidade de área plantada de arroz, se encontra no ranking das sete cidades mais produtivas nesse setor na região.

Tanto em termos econômicos como sociais, o arroz apresenta grande valor em âmbito mundial por ser um dos melhores alimentos para a nutrição humana (Capitani; Miranda; Filho, 2011). A globalização econômica, por sua vez, atinge todos os círculos da sociedade, exigindo que o agricultor invista em capacitação e profissionalismo gerencial e que tenha um forte compromisso com a qualidade da produção (EMBRAPA, 2014b).

Frente ao cenário previamente comentado, o estudo da viabilidade de um investimento, por meio da Engenharia Econômica, consiste em uma atividade necessária no intuito de auxiliar a tomada de decisão. Hirschfeld, (2010) comenta que, esse estudo de viabilidade consiste em uma série de aspectos, como a análise do projeto, com a finalidade de verificar pontos financeiros, jurídicos, competitivos, comerciais e técnicos.

$\mathrm{O}$ presente estudo objetiva analisar a viabilidade de produção de arroz irrigado na cidade de Agudo - RS, para médios e pequenos agricultores. Fazendo uso de métodos que compõem o escopo da Engenharia econômica, o objetivo deste estudo é conduzido pelo método de Valor Presente Líquido (VPL) e pelo estudo de tempo de retorno de investimento (Payback descontado).

\section{ENGENHARIA ECONÔMICA}

Alternativas econômicas são analisadas de acordo com algum critério econômico pré-determinado, que levam a decisão de distribuição dos recursos em alguma das alternativas econômicas (Hirschfeld, 2010). Na concepção de Casarotto Filho e Kopittke (2010), o uso das técnicas de engenharia econômica auxilia na análise de investimentos, que engloba desde critérios somente monetários até observações mais complexas, de vantagem competitiva a impactos sociais e ambientais.

Casarotto Filho e Kopittke (2010) e Schrippe, Bortolotti e Possan (2012) declaram que a engenharia econômica possui os seguintes três métodos básicos para a análise de investimentos: (1) Valor Anual Uniforme Equivalente (VAUE); (2) Valor Presente Líquido (VPL); e (3) Taxa Interna de Retorno (TIR). Esses métodos, se aplicados corretamente, trazem os mesmos resultados de análise.

\subsection{VALOR PRESENTE LÍQUIDO}

O Método do Valor Presente Líquido determina um valor no momento que é trazido como inicial, com base de um fluxo de caixa formado com uma sequência de benefícios e custos descontados a uma Taxa de Mínima Atratividade (TMA) do investidor (Motta; Calôba, 2009; Hirschfeld, 2010). Na compreensão de Rossi et al. (2010), o VPL é importante quando é desejado comparar opções de investimentos mutuamente excludentes e então são trazidos ao âmbito atual todos os custos e ganhos do período. A opção que trouxer o maior valor presente líquido é a escolhida. Como visto, o método do VPL mede o aumento da riqueza líquida no 
momento em que seria equivalente à implantação do projeto.

Ao analisar um possível investimento, é necessário considerar que o capital investido não irá mais render em outro tipo de aplicação, geralmente, tendo em base a cultura brasileira, é de praxe a utilização da taxa de caderneta de poupança. Cherobim e Espejo (2010) corroboram que a tradicional caderneta de poupança da Caixa Econômica Federal lidera o ranking das aplicações financeiras das famílias no país.

Todavia, em situações mais robustas, é sugerida a utilização de modelos como o Capital Asset Pricing Model (CAPM) desenvolvido por Sharpe (1964) e Lintner (1965), que de acordo com Guermat (2014) CAPM possui uma relação linear entre o $\beta$ (beta) e o valor esperado. Outro modelo possível de utilização seria o desenvolvido por Ross (1976) o Arbitrage Pricing Theory (APT), analisado por Zabarankin, Pavlikov e Uryasev (2014) como modelo que explica os retornos de ativos por meio de modelos lineares com vários fatores, cada qual com o seu $\beta$, sendo considerado um melhoramento do CAPM.

Doravante, a TMA do investimento deve ser no mínimo igual à taxa de juros equivalente a rentabilidade do capital em aplicações correntes (Casarotto Filho; Kopittke, 2010). Dessa maneira, Schroeder et al. (2005), Pasqual, Padilla e Jadotte (2013) e Espinoza (2014) reforçam que, quando o VPL de um investimento se mostrar superior a zero o método sugere do ponto de vista econômico-financeiro que o investimento deve ser feito, porque além de bater os custos do projeto, gera um retorno adicional. Casarotto Filho e Kopittke (2010) apontam a Equação 1 que esquematiza a obtenção do VPL.

$$
V P L=\sum_{t=1}^{n}\left(\frac{F C_{t}}{(1+k)^{t}}\right)-F C_{0}
$$

Sendo que: VPL: referente a um fluxo de caixa de um investimento, [R $\$]$; n: Número de períodos na linha do tempo, [períodos]; t: Período genérico na linha do tempo, [ano]; k: Taxa de custo de capital da empresa, TMA, [\% a.a.]; $\mathrm{FC}_{0}$ : Valor inicial do investimento, [R\$]; e $\mathrm{FC}_{\mathrm{t}}$ : Valores futuros das entradas e/ou saídas de caixa a cada data "t", [R\$].

$\mathrm{Em}$ relação às vantagens $\mathrm{e}$ desvantagens do método VPL, Lemes Júnior, Rigo e Cherobim (2005) apontam, conforme Tabela 1.

Tabela 1. Vantagens e desvantagens do método VPL

\begin{tabular}{|l|l|}
\hline Vantagens & Desvantagens \\
\hline $\begin{array}{l}\text { Considera o valor do } \\
\text { dinheiro no tempo; }\end{array}$ & $\begin{array}{l}\text { Sujeito à determinação } \\
\text { do custo do capital }\end{array}$ \\
\cline { 1 - 2 } $\begin{array}{l}\text { Os VPLs podem ser } \\
\text { somados; }\end{array}$ & $\begin{array}{l}\text { Conceito de difícil } \\
\text { assimilação pelos } \\
\text { empresários em relação }\end{array}$ \\
\cline { 1 - 2 } $\begin{array}{l}\text { Dependem apenas dos } \\
\text { fluxos de caixa e do } \\
\text { custo de capital. }\end{array}$ & à TIR \\
\hline
\end{tabular}

Fonte: Lemes Júnior, Rigo e Cherobim (2005)

Diante dos aspectos mencionados na Tabela 1, o VPL se mostra um método de análise de investimentos que traz resultados pertinentes e seguros ao estudo em questão.

\subsection{PAYBACK DESCONTADO}

Payback, ou tempo de recuperação do capital, consiste na mensuração do número de anos requeridos para a recuperação de um dado investimento inicial, ou seja, este método mede o tempo necessário para que a soma das quantias anuais seja igual ao investimento original (Casarotto; Kopittke, 2010). Por ser um método de análise de investimentos não exato, deve ser usado apenas como segundo dado de análise, ou em avaliações de investimentos de lucro puro.

Heysel e Filion (2013) reforçam que, como o Payback é a mensuração da quantidade de tempo que o projeto leva para ter seu custo recuperado totalmente, é sempre desejado um menor valor possível de Payback para o investimento a ser realizado. Lemes Junior, Rigo e Cherobim (2005) apontam que as vantagens de se utilizar o método do Payback são: o fácil entendimento, o fato de favorecer a liquidez e também o caso 
de considerar a incerteza de fluxos de caixa distantes. Mas uma grande desvantagem deste método é que ele não considera o valor do dinheiro no tempo nem os fluxos de caixa pós Payback.

No intuito de obter os benefícios do método Payback, considerando o valor do dinheiro no tempo, foi desenvolvimento o Payback descontado, que remete a uma projeção do tempo necessário para o projeto se pagar, considerando a TMA do investidor (KASSAI et al., 2007). As vantagens e desvantagens do método Payback descontado estão apontados na Tabela 2.

Tabela 2. Payback descontado

\begin{tabular}{|c|c|}
\hline Vantagens & Desvantagens \\
\hline 1. Fácil de entender & $\begin{array}{l}\text { 1. Exige um período } \\
\text { limite arbitrário }\end{array}$ \\
\hline 2. Favorece a liquidez & $\begin{array}{l}\text { 2. Penaliza projetos de } \\
\text { longo prazo }\end{array}$ \\
\hline $\begin{array}{l}\text { 3. Considera o valor do } \\
\text { dinheiro no tempo }\end{array}$ & 3. Ignora fluxos de \\
\hline $\begin{array}{l}\text { 4. Considera a } \\
\text { incerteza de fluxo de } \\
\text { caixa mais distante }\end{array}$ & $\begin{array}{l}\text { caixa pós Payback } \\
\text { descontado }\end{array}$ \\
\hline
\end{tabular}

Fonte: Lemes Júnior, Rigo e Cherobim (2005)

Tendo como base a Tabela 2 é possível compreender que utilizar apenas o Payback descontado pode ser perigoso. Ainda que, o Payback descontado apresente vantagens consideráveis, é interessante que o mesmo seja utilizado como método auxiliar a um VPL ou TIR para uma tomada de decisão.

\section{METODOLOGIA}

A pesquisa se encontra esquematizada conforme a Figura 1.

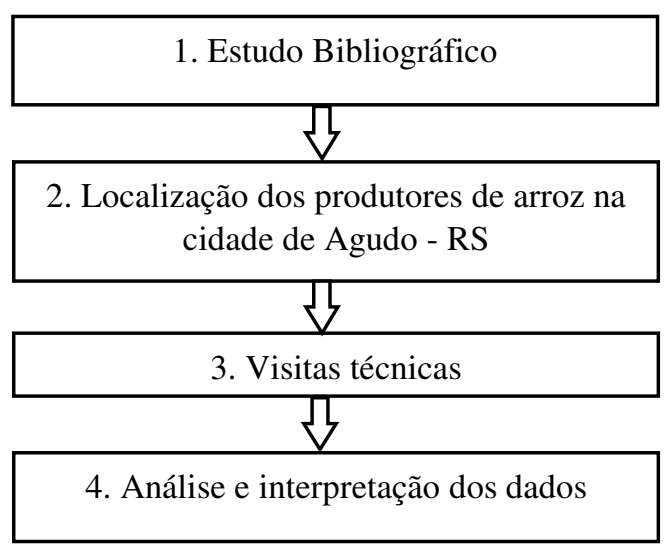

Figura 1 - Etapas da pesquisa
Em relação à Figura 1, na primeira etapa, foi realizado $o$ embasamento do estudo por meio de referências teóricas publicadas em livros, jornais, artigos etc. Na segunda etapa, foi realizado contato com empresas $\mathrm{e}$ cooperativas de arroz da região e as prefeituras das cidades da Quarta Colônia, com a finalidade de definir o objeto de estudo.

Segundo dados do Instituto Rio Grandense do Arroz (IRGA, 2014), a região de Agudo possui uma grande quantidade de hectares destinados unicamente à produção de $\operatorname{arroz} \mathrm{e}$, historicamente, por apresentar terras favoráveis ao seu cultivo, exibe uma alta produtividade. Dessa maneira, o estudo de caso foi realizado em uma propriedade rural localizada entre as cidades de Dona Francisca e Agudo, região da Quarta Colônia, no Rio Grande do Sul, situada a aproximadamente $240 \mathrm{~km}$ da Capital do Estado (Porto Alegre).

Por meio de visitas técnicas foi possível acompanhar as etapas de produção e levantar todos os dados necessários para o presente trabalho, tais como: os equipamentos necessários para a produção, observação dos empecilhos de produção e os custos por hectare de arroz plantado. Na quarta e última etapa, se contabilizaram os custos da produção de arroz, e então, fez-se o estudo da viabilidade técnica e econômica de se produzir o arroz, levando em consideração todo o investimento em equipamentos e insumos necessários. A construção desta pesquisa realizou-se entre março de 2013 e abril de 2014.

\section{RESULTADOS E DISCUSSÃO}

Para estimar a viabilidade técnica e econômica do projeto, quanto ao maquinário, os dados necessários foram coletados com um produtor da cidade de Agudo - RS, sendo que este possui 50 hectares destinados à produção de arroz. Inicialmente, foram levantados os investimentos necessários, que se encontram dispostos na Tabela 3. 
Tabela 3. Investimento em maquinário

\begin{tabular}{|l|l|}
\hline \multicolumn{1}{|c|}{ Item e quantidade } & \multicolumn{1}{c|}{ Valor total $(\mathbf{R} \mathbf{\text { ) }}$} \\
\hline 2 Tratores tracionados & $280.000,00$ \\
\hline 1 Trator articulado & $140.000,00$ \\
\hline 1 Colheitadeira & $350.000,00$ \\
\hline 2 Silos & $240.000,00$ \\
\hline TOTAL & $\mathbf{1 . 0 1 0 . 0 0 0 , 0 0}$ \\
\hline
\end{tabular}

Considerando a Tabela 3, visualiza-se que a propriedade em questão necessita de 2 tratores tracionados de potência de $100 \mathrm{cv}, 1$ trator articulado, 1 colheitadeira de tamanho médio e 2 silos que suportem 50.000 sacos de $50 \mathrm{~kg}$ cada. Os valores unitários do maquinário são referentes à aquisição desses equipamentos no ano de 2014, em empresas conceituadas da região. Então, os investimentos necessários de maquinário para a propriedade estudada totalizam $\mathrm{R} \$ 1.010 .000,00$.

$\mathrm{Na}$ sequência, foram contabilizados os dispêndios relacionados à plantação do arroz. $\mathrm{O}$ modo de plantio utilizado pelo produtor é o modo prégeminado, que consiste em lançar sementes pré-geminadas em um campo totalmente inundado. Com isso, são identificadas as principais etapas de produção e a soma dos custos de cada etapa. A Tabela 4 dispõe os custos referentes à produção de arroz.

Tabela 4. Custos para produzir

\begin{tabular}{|l|c|}
\hline Item & Valor Total \\
\hline Dessecação do Solo & $\mathrm{R} \$ 4.650,00$ \\
\hline Preparo do Solo & $\mathrm{R} \$ 3.100,00$ \\
\hline Semeadura & $\mathrm{R} \$ 11.600,00$ \\
\hline Adubação & $\mathrm{R} \$ 36.750,00$ \\
\hline $\begin{array}{l}\text { Controle de plantas } \\
\text { daninhas }\end{array}$ & $\mathrm{R} \$ 14.700,00$ \\
\hline Controle de pragas & $\mathrm{R} \$ 11.750,00$ \\
\hline Irrigação & $\mathrm{R} \$ 18.000,00$ \\
\hline $\begin{array}{l}\text { Colheita, transporte } \mathrm{e} \\
\text { secagem }\end{array}$ & $\mathrm{R} \$ 41.055,00$ \\
\hline Total & $\mathrm{R} \$ 141.605,00$ \\
\hline
\end{tabular}

Os dados da Tabela 4 dizem respeito à safra 2013/2014 e todos os valores foram disponibilizados em relação à área de 50 hectares de produção de arroz. Incluso nesses dados foram considerados: todos os insumos utilizados para a produção, o combustível gasto em cada uma das etapas e o custo com energia elétrica para as bombas de irrigação.

E mais detalhadamente, na seção "Colheita, transporte e secagem" é considerada uma produtividade média de $7.000 \mathrm{~kg} / \mathrm{ha}$ e então calculadas a percentagem que corresponde: à colheita $(8 \%)$, ao transporte $(3 \%)$ e à secagem $(6 \%)$.

As taxas de depreciação utilizadas nos cálculos dos métodos de análise de investimentos foram de $25 \%$ a.a para veículos (tratores) e de $10 \%$ a.a para equipamentos em geral (silos). Foi considerado que todos esses equipamentos seriam adquiridos apenas uma vez no tempo zero e, após o processo de depreciação, todos os equipamentos não possuíram valor comercial. Foi estimado, portanto, a vida útil dos equipamentos maior do que a vida contábil.

Para a entrada do fluxo de caixa do projeto foi considerada a média de produtividade de arroz da região do centro do Estado do Rio Grande do Sul, que é de $7.000 \mathrm{~kg} / \mathrm{ha}$, com isso, cada hectare produz 140 sacos de arroz de 50 $\mathrm{kg}$ cada. Para o valor de venda de cada saco de arroz foi utilizada a média de venda do ano de 2013, este valor é de R\$ 34,50 . Por fim, o valor obtido com a venda da safra é de R\$241.500,00.

Com a apuração das entradas e saídas do estudo realizado e, considerando a depreciação do maquinário de acordo com as normas, foi possível obter o cálculo do fluxo de caixa dos anos que se seguirão (Tabela 5).

É importante ressaltar que neste fluxo de caixa não são consideradas as variações dos preços de plantio do arroz no decorrer dos anos, bem como o valor de venda do saco de arroz. Também se considera que o maquinário foi adquirido somente uma vez em toda a análise e que o Imposto de Renda (IR) é de $8,28 \%$ ao ano.

$\mathrm{Na}$ Tabela 5 é possível observar o tempo que o caixa leva para tornar positivo o projeto. Considerando as suas entradas e saídas, bem como uma TMA de $6,41 \%$ a.a. - taxa de poupança no ano de 2012 de acordo com o Banco Central 
do Brasil (2014) - visualizou-se que para o período de 25 anos ainda não teríamos um retorno do investimento utilizando o Payback descontado, assim, inviabilizando o projeto.

Tabela 5. Fluxo de caixa

\begin{tabular}{|l|l|l|}
\hline Ano & Fluxo de caixa & \multicolumn{1}{|c|}{$\begin{array}{c}\text { Fluxo de caixa } \\
\text { acumulado } \\
\text { descontado }\end{array}$} \\
\hline 0 & $-\mathrm{R} \$ 1.151 .605,00$ & $-\mathrm{R} \$ 1.151 .605,00$ \\
\hline 1 & $-\mathrm{R} \$ 116.605,00$ & $-\mathrm{R} \$ 1.342 .062,43$ \\
\hline 2 & $-\mathrm{R} \$ 116.605,00$ & $-\mathrm{R} \$ 1.544 .733,89$ \\
\hline 3 & $-\mathrm{R} \$ 116.605,00$ & $-\mathrm{R} \$ 1.760 .402,68$ \\
\hline 4 & $-\mathrm{R} \$ 116.605,00$ & $-\mathrm{R} \$ 1.989 .902,30$ \\
\hline 5 & $\mathrm{R} \$ 69.610,89$ & $-\mathrm{R} \$ 2.047 .903,84$ \\
\hline 6 & $\mathrm{R} \$ 69.610,89$ & $-\mathrm{R} \$ 2.109 .625,02$ \\
\hline 7 & $\mathrm{R} \$ 69.610,89$ & $-\mathrm{R} \$ 2.175 .304,38$ \\
\hline 8 & $\mathrm{R} \$ 69.610,89$ & $-\mathrm{R} \$ 2.245 .195,75$ \\
\hline 9 & $\mathrm{R} \$ 69.610,89$ & $-\mathrm{R} \$ 2.319 .569,26$ \\
\hline 10 & $\mathrm{R} \$ 69.610,89$ & $-\mathrm{R} \$ 2.398 .712,35$ \\
\hline 11 & $\mathrm{R} \$ 91.623,69$ & $-\mathrm{R} \$ 2.460 .918,08$ \\
\hline 12 & $\mathrm{R} \$ 91.623,69$ & $-\mathrm{R} \$ 2.527 .113,06$ \\
\hline 13 & $\mathrm{R} \$ 91.623,69$ & $-\mathrm{R} \$ 2.597 .553,12$ \\
\hline 14 & $\mathrm{R} \$ 91.623,69$ & $-\mathrm{R} \$ 2.672 .510,51$ \\
\hline 15 & $\mathrm{R} \$ 91.623,69$ & $-\mathrm{R} \$ 2.752 .274,92$ \\
\hline 16 & $\mathrm{R} \$ 91.623,69$ & $-\mathrm{R} \$ 2.837 .154,61$ \\
\hline 17 & $\mathrm{R} \$ 91.623,69$ & $-\mathrm{R} \$ 2.927 .477,65$ \\
\hline 18 & $\mathrm{R} \$ 91.623,69$ & $-\mathrm{R} \$ 3.023 .593,09$ \\
\hline 19 & $\mathrm{R} \$ 91.623,69$ & $-\mathrm{R} \$ 3.125 .872,42$ \\
\hline 20 & $\mathrm{R} \$ 91.623,69$ & $-\mathrm{R} \$ 3.234 .710,93$ \\
\hline 21 & $\mathrm{R} \$ 91.623,69$ & $-\mathrm{R} \$ 3.350 .529,25$ \\
\hline 22 & $\mathrm{R} \$ 91.623,69$ & $-\mathrm{R} \$ 3.473 .774,99$ \\
\hline 23 & $\mathrm{R} \$ 91.623,69$ & $-\mathrm{R} \$ 3.604 .924,49$ \\
\hline 24 & $\mathrm{R} \$ 91.623,69$ & $-\mathrm{R} \$ 3.744 .484,60$ \\
\hline 25 & $\mathrm{R} \$ 91.623,69$ & $-\mathrm{R} \$ 3.892 .994,71$ \\
\hline & &
\end{tabular}

Complementarmente, a análise desconsiderou o fato de que qualquer intempérie pode causar consideráveis perdas, o que poderia alterar drasticamente os indicadores.

Sequencialmente, considerando uma TMA do proprietário de $6,41 \%$ a.a., bem como dos fluxos do projeto, foi obtido um VPL de - R\$ 892.402,33 (oitocentos e noventa e dois mil quatrocentos e dois reais e trinta e três centavos negativos). Portanto, considerando as entradas e dispêndios do projeto no tempo, descontados a TMA, obteve-se um VPL negativo no tempo zero. Dessa forma, o método VPL aponta inviabilidade econômica do projeto, caso esse fosse implantado no momento.

\section{CONSIDERAÇÕES FINAIS}

$\mathrm{O}$ pequeno e o médio produtor enfrentam sérias dificuldades em relação aos custos de produção, principalmente oriundas da dependência de outros serviços, tais como: aquisição de insumos, transporte, secagem em silos e os altos preços dos implementos agrícolas. Tendo em foco este cenário, o presente estudo se concentrou em atender ao objetivo de estimar a viabilidade de produção de arroz irrigado.

Salienta-se que, a fim de facilitar os cálculos, foi desconsiderada uma série de aspectos que podem impactar nos resultados, como no caso: o valor da aquisição da terra necessária para o plantio, histórico e possibilidade de perdas devida a intempéries de produção, variação dos valores de venda do arroz e uma série de impostos, haja vista que foi considerado apenas o IR de 8,28\%.

A inviabilidade do projeto foi constatada pelo método do VPL que resultou em - R \$ 892.402,33. Complementarmente, o Payback descontado aponta que em um período de 25 anos não se obterá retorno do investimento. Ainda que desconsiderados uma série de fatores que tendem a encarecer o processo, o estudo apontou que o investimento não é economicamente interessante.

Entretanto, a atividade produtiva do arroz apresenta grande valor para os vários círculos da sociedade, desde os agricultores até os consumidores finais do alimento, visto que o arroz é o grande protagonista da refeição diária do brasileiro. Diante disso, é importante a participação de incentivos governamentais que garantam que a produção de arroz seja possível e segura para estes produtores.

Complementarmente, no intuito de sanar esse problema, a União Europeia (UE) e os Estados Unidos da América (EUA) podem ser usados como uma referência de organização política na agricultura. A agricultura na União Europeia é organizada pela Política Agrícola Comum (PAC) que tem por objetivo assegurar um nível de vida justo 
para os agricultores e preços acessíveis aos consumidores. Nos EUA a organização da agricultura é realizada de acordo com o solo e o clima, chamados de cinturões. Ambos possuem políticas que respeitam tanto os consumidores como os agricultores, o que ajuda no desenvolvimento de todos os setores, sendo que a agricultura é o setor primário, e então, fundamental para a sociedade.

O trabalho aponta uma série de lacunas para estudos, tanto na área de custos, como na área das ciências rurais e sociais, tais como: (1) a própria análise de viabilidade técnico-econômica, foi excessivamente otimista e concentrada na realidade de apenas uma propriedade, um estudo mais amplo, seria interessante; (2) simulação da área de produção, supondo possíveis produtividades por hectare e o quanto isso reflete na viabilidade de se produzir arroz; (3) análise das atividades agronômicas, bem como o desenvolvimento de um melhor método de plantio; e (4) o estudo de políticas públicas, desenvolvendo pesquisas sociais para possíveis subsídios aos agricultores que tiverem algum prejuízo.

\section{REFERÊNCIAS}

BANCO CENTRAL DO BRASIL. Remuneração dos depósitos de poupança. 2014. Disponível em: $<$ http://www4.bcb.gov.br/pec/poupanca/p oupanca.asp>. Acesso em: 08/10/2014.

CAPITANI, D. H. D., MIRANDA, S. H. G., FILHO, J. G. M. 2011. Determinantes da demanda brasileira por importação de arroz do Mercosul. Revista de Economia e Sociologia Rural, 49, 545-572.

CASAROTTO FILHO, N., KOPITTKE, B. H. 2010. Análise de investimentos. 11. ed. São Paulo, Atlas.

CHEROBIM, A. P. M. S.; ESPEJO, M. M. dos S. B. (Orgs.). 2010. Finanças pessoais: conhecer para enriquecer! São Paulo, Atlas.
DIÁRIO DE SANTA MARIA. O arroz em ascensão. Diário de Santa Maria, Santa Maria, 27 maio 2013. Caderno economia, p. 5.

EMBRAPA. Cultivo de arroz irrigado no $R S, \quad 2014$ a. Disponível em: $<$ http://sistemasdeproducao.cnptia.embra pa.br/FontesHTML/Arroz/ArrozIrrigado Brasil/cap01.htm>. Acesso em: 13/03/2014.

EMBRAPA. A criação do gado leiteiro na zona Bragantina, 2014b. Disponível em: <http://sistemasdeproducao.cnptia. embrapa.br/FontesHTML/Leite/GadoLeit eiroZonaBragantina/paginas/cadeia.htm> Acesso em: 4/05/2014.

ESPINOZA, R. D. 2014. Separating project risk from the time value of money: a step toward integration of risk management and valuation of infrastructure investments. International Journal of Project Management, 32, 6, 1056-1072.

GELATTI, J. No caminho de uma boa safra. Diário de Santa Maria, Santa Maria, 8 mar. 2014. Caderno economia, p. 10.

GUERMAT, C. 2014. Yes, the CAPM is testable. Journal of Banking \& Finance, 46, 31-42.

HEYSEL, C. S, FILION, Y. R. 2013. Estimating the payback period of in-line micro turbines with analytical probabilistic models. Procedia Engineering, 70, 815-822.

HIRSCHFELD, H. 2010. Engenharia econômica e análise de custos. São Paulo, Atlas.

INSTITUTO RIO GRANDENSE DO ARROZ - IRGA. Produtividades municipais safra 2012/13. Disponível em:

<http://www3.irga.rs.gov.br/uploads/anex os/1374499016SAFRA_2012_13_RS_M UNICIPAL.pdf $>$. Acesso em: 14/03/2014. 
KASSAI, J. R.; KASSAI, S.; SANTOS, A.; ASSAF NETO, A. 2007. Retorno de investimento: abordagens matemática $\mathrm{e}$ contábil do lucro empresarial. São Paulo, Atlas.

LEMES JÚNIOR, A. B., RIGO, C. M., CHEROBIM, A. P. 2005. Administração financeira: princípios, fundamentos $\mathrm{e}$ práticas brasileiras. 2. ed. Rio de Janeiro, Campus.

LINTNER, J. 1965. The valuation of risk assets and the selection of risky investments in stock portfolios and capital budgets. The Review of Economics and Statistics, 47, 1, 13-37.

MOTTA, R. R., CALÔBA, G. M. 2009. Análise de investimentos: tomada de decisão em projetos industriais. São Paulo, Atlas.

PASQUAL, J., PADILLA, E., JADOTTE, E. 2013. Technical note: equivalence of different profitability criteria with the net present value. International Journal of Production Economics, 142, 205-210.

ROSS, S. 1976. The arbitrage theory of capital asset pricing. Journal of Economic Theory, 13, 341-60.

ROSSI, L. A., DAVID, E., SARUBBI, J., PAGLIARDI, O. 2010. Viabilidade técnico-econômica do uso de diferentes tecnologias de iluminação para indução de fotoperíodo na produção de mudas de crisântemo. Engenharia Agrícola, Jaboticabal, 30, 1-8.

SHARPE, W. F. 1964. Capital asset prices: a theory of market equilibrium under conditions of risk. Journal of Finance, 9, 425-442.

SCHRIPPE, P.; BORTOLOTTI, S. L. V.; POSSAN, E. Estudo da viabilidade técnico-econômica da recuperação da fécula em uma fecularia de mandioca. In: ENCONTRO NACIONAL DE ENGENHARIA DE PRODUÇÃO, 32, 2012, Bento Gonçalves. Anais... Rio de
Janeiro: Associação Brasileira de Engenharia de Produção. 1-9.

SCHROEDER, J. T.; SCHROEDER, I.; COSTA, R. P.; SHINODA, C. 2005. O Custo de capital como taxa mínima de atratividade na avaliação de projetos de investimento. Revista Gestão Industrial, $1,36-45$.

ZABARANKIN, M.; PAVLIKOV, K.; URYASEV, S. 2014. Capital Asset Pricing Model (CAPM) with drawdown measure. European Journal of Operational Research, 234, 2, 508-517. 